\title{
Exaustão emocional: relações com a percepção de suporte organizacional e com as estratégias de coping no trabalho
}

\author{
Mauricio Robayo Tamayo \\ Bartholomeu Tôrres Tróccoli \\ Universidade de Brasília
}

\begin{abstract}
Resumo
A exaustão emocional é considerada como o fator central do burnout. Diversas pesquisas mostram que características do ambiente de trabalho e do trabalhador estão associadas ao desenvolvimento da exaustão emocional. Este trabalho apresenta os resultados de uma investigação sobre dois aspectos importantes para o estudo da exaustão emocional: a percepção de suporte organizacional (PSO) e o coping no trabalho. 369 trabalhadores responderam uma escala de exaustão emocional, uma de PSO e outra de coping no trabalho. Os fatores gestão de desempenho, sobrecarga, suporte social e ascensão e salários da escala de PSO e o fator escape da escala de coping revelaram-se preditores significativos da dimensão exaustão psicológica da escala de exaustão emocional. A segunda dimensão desta escala, percepção de desgaste, só foi predita significativamente pelos fatores suporte social e sobrecarga da escala de PSO. Recomendações para lidar com a exaustão emocional são apresentadas.
\end{abstract}

Palavras-Chave: Exaustão emocional, Burnout, Suporte organizacional, Coping.

\begin{abstract}
Emotional exhaustion: Relationships with the perceived organizational support and coping strategies in the work place

Emotional exhaustion is regarded as the principal dimension of the burnout. Several studies show that characteristics of job setting and the employee are associated with the development of emotional exhaustion. This study present the results of a research about the relationship among emotional exhaustion, the perceived organizational support (POS) and coping strategies in the work place. 396 workers answered a scale of emotional exhaustion, one of POS and one another of coping. The dimensions performance management, overload, social support and promotion and salaries from the POS scale and the dimension escape from the coping scale were significant predictors of the dimension psychological exhaustion from the emotional exhaustion scale. The second dimension from the emotional exhaustion scale, perception of wear, was significantly predicted only by the factors social support and overload from the POS scale. Recommendations for the management of emotional exhaustion are presented.
\end{abstract}

Key-Words: Emotional exhaustion, Burnout, Organizational support, Coping.

$\mathrm{O}$ burnout (esgotamento profissional) é definido como uma síndrome psicológica decorrente da tensão emocional crônica no trabalho. Trata-se de uma experiência subjetiva interna que gera sentimentos e atitudes negativas no relacionamento do indivíduo com o seu trabalho (insatisfação, desgaste, perda do comprometimento), minando o seu desempenho profissional e trazendo conse- qüências indesejáveis para a organização (absenteísmo, abandono do emprego, baixa produtividade). O burnout é caracterizado pelas dimensões exaustão emocional, despersonalização e diminuição da realização pessoal.

A exaustão emocional é considerada pela literatura como a primeira etapa e o fator central da síndrome de burnout (Cordes \& Dougherty, 1993; Gaines \& Jermier, 
1983; Maslach \& Jackson, 1986; Maslach, 1993). Devido a sua natureza afetiva e as características que compartilha com outros estados de fadiga crônica, a exaustão emocional pode apresentar-se em diferentes tipos de categorias ocupacionais que lidam com condições de trabalho estressantes (Gaines \& Jermier, 1983). A exaustão emocional é definida como uma resposta ao estresse ocupacional crônico, caracterizada por sentimentos de desgaste físico e emocional. O indivíduo sente que está sendo super exigido e reduzido nos seus recursos emocionais (Maslach \& Jackson 1986; Maslach, 1993).

Para Cordes e Dougherty (1993) e Zohar (1997), a exaustão emocional é, primordialmente, uma resposta às demandas dos estressores que os empregados devem enfrentar, tais como a sobrecarga de trabalho, os contatos interpessoais, o papel conflituoso e os altos níveis de expectativas do indivíduo com relação a si próprio e a sua organização. A exaustão emocional reflete, portanto, os efeitos de demandas organizacionais e individuais (Cordes \& Dougherty, 1993). A exaustão emocional pode comprometer a saúde mental e física dos trabalhadores e deteriorar a qualidade de vida no trabalho e o funcionamento da organização (Wright \& Cropanzano, 1998).

Diversos estudos têm abordado a relação entre a exaustão emocional e variáveis da organização e do traba1ho. Na sua meta-análise dos correlatos das três dimensões do burnout, Lee e Asforth (1996), por exemplo, examinaram 61 pesquisas. Os resultados revelaram correlações altas e moderadas entre a exaustão emocional e variáveis organizacionais e do trabalho. Por um lado, a exaustão emocional correlacionou-se positivamente com o papel conflituoso, a sobrecarga, a pressão no trabalho e o estresse ocupacional. Por outro lado, correlacionou-se negativamente com o suporte social proveniente de fontes externas ao ambiente laboral (cônjuge, amigos, parentes), o suporte do supervisor, as expectativas não atingidas pelo trabalhador e a inovação e a participação no trabalho.

Estudos posteriores continuaram investigando a relação da exaustão emocional com aspectos do ambiente de trabalho percebidos pelo indivíduo e com características da organização. Wright e Bonnet (1997) realizaram uma pesquisa longitudinal com uma amostra de 44 funcionários de várias agências governamentais que trabalhavam em tarefas de prestação de serviços e de supervisão. Os resultados indicaram uma correlação inversa entre a exaustão emocional e o desempenho no trabalho.

A mesma relação inversa entre exaustão emocional e desempenho no trabalho também foi observada em outro estudo longitudinal realizado com uma amostra de 52 assistentes sociais (Wright \& Cropanzano, 1998). Além do desempenho no trabalho, este estudo também investigou a re- lação entre exaustão emocional, satisfação no trabalho e rotatividade voluntária. Os resultados apontaram uma relação direta entre exaustão e rotatividade voluntária e uma relação inversa entre exaustão e satisfação no trabalho.

O papel ambíguo e conflituoso e a sobrecarga no trabalho também foram identificados como preditores da exaustão emocional em uma amostra de enfermeiros (Zohar, 1997) e em uma amostra de vendedores (Babakus, Cravens, Johnston \& Moncrief, 1999). Neste último estudo, a variável exaustão emocional também foi considerada como fator de mediação entre o papel ambíguo e o papel conflituoso, o comprometimento organizacional, a satisfação e o desempenho no trabalho e a intenção de deixar a organização.

No Brasil, alguns estudos têm apontado para a associação entre a exaustão emocional com variáveis da organização, da natureza do trabalho e do indivíduo. Tamayo (1997) verificou, em uma amostra de 229 enfermeiros, que os valores organizacionais de autonomia, conservação, estrutura igualitária e harmonia são preditores significativos da exaustão emocional. Outros autores evidenciaram que o tipo de profissão (dentista e controlador de vôo) e as prioridades axiológicas do indivíduo (tipos motivacionais de valores: tradição, poder, estimulação e benevolência) também são preditores significativos da exaustão emocional (Tamayo et al., 2000).

Os resultados dessas pesquisas e de outros estudos sobre burnout e exaustão emocional têm apontado para a importância de investigar variáveis envolvidas na transação entre o indivíduo e o seu ambiente laboral. Duas dessas variáveis são o suporte organizacional percebido e as estratégias de coping utilizadas pelo trabalhador para lidar com o estresse ocupacional.

\section{Percepção de Suporte Organizacional (PSO)}

As crenças e as expectativas do indivíduo acerca da retribuição e do reconhecimento dado pela organização ao seu trabalho são questões diretamente relacionadas à percepção de suporte organizacional. Este constructo foi definido por Eisenberger, Huntington, Hutchison e Sowa (1986) como "as crenças globais desenvolvidas pelo empregado sobre a extensão em que a organização valoriza as suas contribuições e cuida do seu bem-estar" (p. 501).

A percepção de suporte organizacional depende do mesmo processo de atribuição que as pessoas utilizam, geralmente, para definir o comprometimento dos outros nas relações sociais (Eisenberger, Huntington, Hutchison \& Sowa, 1986). Essa percepção é influenciada pela freqüência, intensidade e sinceridade das expressões de elogio e aprovação e, também, pelos aspectos ligados ao pagamento, à categoria do trabalho, ao enriquecimento do trabalho e à influência sobre as políticas da organização 
(Blau, 1964; Brinberg \& Castell, 1982, citados por Eisenberger et al., 1986).

Segundo Cordes e Dougherty (1993), altas expectativas ou expectativas não atingidas com relação a desafios no trabalho, a recompensas, ao reconhecimento, ao avanço na carreira e a outros aspectos laborais podem gerar estresse e burnout. As expectativas dos empregados acerca da profissão, da organização e da sua própria eficácia pessoal podem ter também uma contribuição significativa para o burnout.

Os empregados entram na organização com certas expectativas sobre o que eles poderão realizar profissionalmente e sobre o que a organização poderá oferecer-lhes como recompensa e para facilitar o seu trabalho. Com o passar do tempo, essas expectativas são contrastadas com a realidade do dia-a-dia. Os indivíduos comparam suas expectativas com a sua vivência na organização. Resultados discrepantes nessa comparação podem influenciar o comportamento das pessoas no trabalho (Cordes \& Dougherty, 1993).

O burnout e, conseqüentemente, a exaustão emocional, apresentam-se quando os indivíduos tem de trabalhar numa situação de desbalanceamento crônico, na qual se demanda mais do que as pessoas podem dar e se oferece menos do que elas precisam (Maslach \& Leiter, 1997).

A relação entre a exaustão emocional e o suporte fornecido pela organização tem sido tratada em alguns estudos sobre burnout. Aiken e Sloane (1997), por exemplo, desenvolveram uma pesquisa com uma amostra de 955 enfermeiras. Esses autores verificaram que aquelas lotadas em unidades especializadas no atendimento a pacientes aidéticos apresentavam menor exaustão emocional e percebiam que o suporte oferecido pela organização permitia-lhes ter maior autonomia e controle sobre seu trabalho. Já as enfermeiras que trabalhavam em unidades hospitalares gerais, lidando com todo tipo de pacientes, inclusive os aidéticos, percebiam menor suporte organizacional e apresentavam maior exaustão emocional.

Van Yperen (1998) investigou se a percepção de equidade na relação de troca empregado-empregador estava relacionada ao suporte informacional da organização e ao burnout e se as crenças de auto-eficácia desempenhavam algum papel nessa relação. Os resultados observados em uma amostra de 114 enfermeiras de uma instituição de maternidade indicaram que um maior índice de exaustão emocional estava associado a um menor suporte informacional por parte da organização e a uma menor percepção de equidade. Também foi observado que o vínculo entre o suporte informacional e os sintomas de exaustão emocional foi mediado pela percepção de eqüidade na relação de troca com a organização.

Jayaratne, Himle e Chess (1988) estudaram a relação entre a percepção de suporte, o uso do suporte e os seus efeitos sobre o estresse ocupacional, a tensão e as dimensões do burnout. Os resultados observados em uma amostra de 480 assistentes sociais mostraram que uma maior percepção de suporte do supervisor e dos colegas estava relacionada a um índice menor de exaustão emocional.

A mesma correlação inversa entre suporte social e exaustão emocional foi igualmente relatada em um estudo conduzido por Russell, Altmaier e Van Velzen (1987), desenvolvido numa amostra de 600 professores de várias escolas públicas.

Para Eisenberger et al. (1986) e Schein (1982), o valor dado pelos trabalhadores ao suporte organizacional decorre da atribuição de características antropomórficas à organização, considerando que os empregados tendem a ver as ações dos agentes da organização como ações da própria organização. A organização não atua como uma entidade abstrata, ao contrário, ela atua por meio do comportamento individual de membros-chave que ocupam posições de gerência ou de liderança.

A personificação da organização decorre de vários fatores. Primeiro, a organização tem responsabilidades legais, morais e financeiras pelas ações dos seus agentes. Segundo, precedentes, tradições, políticas e normas organizacionais definem a permanência ou a extinção de certos papéis comportamentais. Por último, a organização, através dos seus agentes, exerce poder sobre cada um dos seus empregados (Levinson, 1965).

\section{Coping no trabalho}

Na definição de Folkman, Lazarus, Dunkel-Schetter, Delongis e Gruen (1986), o coping é compreendido como "esforços cognitivos e comportamentais que mudam constantemente e que se desenvolvem para responder às demandas específicas externas e/ou internas avaliadas como excessivas para os recursos do indivíduo" (p. 993).

Latack e Havlovic (1992) concluíram, após examinar várias definições sobre coping, que existe um acordo sobre a noção de que esse fenômeno é parte de uma transação pessoa-ambiente que acontece quando o indivíduo avalia uma situação como estressante.

Outras definições acentuam diferentes aspectos ou estratégias de enfrentamento ao estresse. Para Zautra e Wrabetz (1991), por exemplo, o coping é um processo dinâmico de esforços determinados para a resolução das dificuldades e das demandas exigidas para o ajustamento do organismo. Para Parkes (1994) e Terry (1994), o coping é um construto multidimensional que envolve uma grande variedade de estratégias cognitivas e comportamentais que podem ser utilizadas para alterar, reavaliar e evitar circunstâncias estressantes ou para aliviar os seus efeitos adversos. 
Para Lazarus e Folkman (1984, citados por Gil-Monte e Peiró, 1997), o coping pode ser compreendido a partir de dois tipos de estratégias: as estratégias focalizadas no problema e as estratégias centradas na emoção. O primeiro tipo tem como objetivo analisar e definir a situação, considerando os seus custos e benefícios, e buscando alternativas para resolvê-la.

Este primeiro tipo inclui estratégias que afetam o ambiente e o sujeito. As estratégias que afetam o ambiente tentam modificar o estressor através de mudanças nas pressões externas, nos obstáculos, nos recursos, nos procedimentos etc. As estratégias que afetam o sujeito incluem mudanças nas aspirações do indivíduo, redução da participação do $E u$, busca de canais de participação alternativos, desenvolvimento de novas pautas de conduta e a aprendizagem de novos procedimentos e recursos.

O segundo tipo de estratégias, sugeridas por Lazarus e Folkman (1984, citados por Gil-Monte \& Peiró, 1997), refere-se aos processos cognitivos responsáveis pela diminuição do transtorno emocional gerado por uma situação estressante. Estas estratégias são utilizadas pelos indivíduos quando percebem que os estressores não podem ser modificados e que é preciso continuar interagindo com eles. O propósito é manter a esperança e o otimismo, negar tanto a situação quanto as suas conseqüências, ou atuar como se não importassem as circunstâncias. Essas estratégias incluem a esquiva, a culpabilidade, o escape, o distanciamento, a atenção seletiva, as comparações positivas e a extração de aspectos positivos de acontecimentos negativos.

De acordo com Koeske, Kirk e Koeske (1993), o estudo das estratégias de coping com o estresse tem uma história mais longa do que com o burnout e, consequentemente, com a exaustão emocional. Após uma revisão de literatura que aborda a relação entre burnout e coping, Gil-Monte e Peiró (1997) concluíram que o uso de estratégias de coping de controle ou centradas no problema previne o desenvolvimento do burnout, enquanto que, a utilização de estratégias de escape, evitação ou centradas na emoção facilita a sua aparição. A disponibilidade de recursos de coping modera a relação entre os estressores e a exaustão emocional (Cordes \& Doughherty, 1993).

Pesquisas relatadas por Thorton (1992) e Etzion e Pines (1986) mostram que as estratégias de coping inativas, tais como o escape, a evitação e a medicação, apresentam uma relação positiva com o burnout. Para esses autores, um nível baixo de burnout permite ao indivíduo enfrentar as situações estressantes de forma ativa e direta, enquanto que, um nível alto de burnout, pode diminuir a energia do su- jeito para lidar com as situações de forma ativa, levandoo a adotar comportamentos passivos e indiretos.

Leiter (1991) realizou uma pesquisa envolvendo 177 trabalhadores de um hospital psiquiátrico (enfermeiras, assistentes sociais, psiquiatras, psicólogos, recreadores, terapeutas ocupacionais). Os resultados do estudo evidenciaram que um índice menor de exaustão emocional estava associado a utilização de estratégias de controle. Também foi verificado que uso de estratégias de escape/ evitação aumenta a exaustão emocional, tornando este tipo de estratégias num meio particularmente ineficaz para evitar o burnout.

Uma crítica comum às medidas de coping geral é que usualmente não levam em conta a importância dos fatores contextuais específicos e, quando o fazem, utilizam episódios de diferentes tipos aos quais as pessoas devem se reportar para identificar a estratégia de coping que utilizariam. Autores como Terry (1994) consideram que a natureza da situação e a forma em que o indivíduo a avalia são determinantes importantes do tipo de estratégia de coping escolhida pelo sujeito para lidar com um evento específico.

Outra crítica refere-se a distância entre os estudos acadêmicos da relação entre coping e burnout e a manifestação desses fenômenos dentro da realidade das organizações (Latack \& Havlovic, 1992). Um aspecto que contribui para esse problema é que a maioria das medidas que avaliam estratégias de coping são aplicáveis a uma variedade de estressores da vida. De acordo com Parkes (1994), no contexto ocupacional pode ser obtida uma melhor compreensão dos processos adaptativos, utilizando instrumentos construídos especificamente para avaliar o coping no trabalho.

Um desses instrumentos é a escala desenvolvida por Latack (1986) para avaliar o coping no ambiente ocupacional. Na opinião de Leiter (1991), esta escala contempla aspectos relevantes tratados na literatura sobre coping, tais como: a dicotomia entre o coping centrado na emoção (escape, esquiva) e o coping centrado no problema (controle) e a ênfase nas avaliações cognitivas e nas ações do indivíduo para administrar a situação.

Para Latack (1986), as estratégias de ação focalizam diretamente o estressor, enquanto que a reavaliação cognitiva está relacionada com a percepção do indivíduo da situação e das emoções que esta lhe produz. Por tal razão, Latack considera que as ações e as reavaliações cognitivas devem estar juntas tanto nas estratégias de enfrentamento (controle) quanto nas estratégias de conteúdo evasivo (escape, evitação).

A abordagem de Latack (1986) e a sua escala compreendem estratégias de coping de controle, escape e manejo de sintomas. Esta escala foi traduzida, adaptada e validada no Brasil e será utilizada no presente estudo, considerando a sua especificidade para abordar o coping no ambiente de 
trabalho e tendo em vista as propriedades psicométricas obtidas na versão brasileira.

Latack e Havlovic (1992) acreditam que as pesquisas sobre coping poderiam reduzir os altos custos do estresse nas organizações. Para estes autores, a necessidade de desenvolver urgentemente estudos de coping no trabalho aparece retratada nas projeções econômicas e no crescimento contínuo de trabalhadores que devem ser compensados por queixas relacionadas ao estresse ocupacional. Este mesmo argumento pode ser usado para ressaltar a importância de pesquisas que abordem a relação entre a exaustão emocional e o coping no contexto ocupacional, desenvolvidas com trabalhadores brasileiros.

O objetivo desta pesquisa foi investigar a relação dos componentes da percepção de suporte organizacional e do coping no trabalho com a exaustão emocional, utilizando escalas multifatoriais especialmente adaptadas para a realidade brasileira.

\section{Método}

\section{Sujeitos}

A amostra final foi constituída por 369 sujeitos de ambos os sexos (190 mulheres e 179 homens), com idade média de 37,3 anos (d.p = 9,79), ocupando diferentes cargos (pesquisadores, escriturários, técnicos, supervisores) em empresas públicas e privadas dos setores bancário, de pesquisa e de serviços, localizadas no Distrito Federal.

\section{Procedimento}

Os sujeitos foram contatados individualmente por seus supervisores e convidados a preencher um questionário auto-aplicado que investigava aspectos da vida no trabalho e que incluía as medidas apresentadas na próxima seção. Foram distribuídos 650 questionários e obteve-se um índice de retorno de $61 \%$, totalizando 396 questionários respondidos.

\section{Medidas}

Escala de Exaustão Emocional. Este instrumento, desenvolvido por Tamayo e Tróccoli (2000) é uma versão modificada da subescala proposta por Maslach e Jackson (1986). A exaustão emocional apresenta as dimensões exaustão psicológica $(\alpha$ de Cronbach $=0,92)$ e percepção de desgaste $(\alpha=0,84)$.

A Exaustão Psicológica é definida como sentimentos de esgotamento e cansaço presentes no indivíduo como conseqüência do seu trabalho. A Percepção de Desgaste é definida como a sensação do indivíduo de que está sendo superexigido no trabalho e levado ao limite da sua resistência.
Escala de Suporte Organizacional Percebido (ESOP). Esta escala foi desenvolvida por Tamayo, Pinheiro, Tróccoli e Paz (2000) e é composta pelos fatores: gestão de desempenho $(\alpha=0,78)$; estilos de gestão da chefia $(\alpha=0,93)$; suporte social no trabalho $(\alpha=0,85)$; sobrecarga $(\alpha=0,81)$; suporte material $(\alpha=0,82)$ e ascensão e salários $(\alpha=0,75)$.

Esses fatores apresentam as seguintes definições:

a - Estilos de Gestão da Chefia - percepção dos indivíduos quanto às formas utilizadas pelo supervisor para traduzir as políticas e práticas organizacionais em sua unidade;

b - Gestão do desempenho - percepção dos indivíduos sobre as políticas e práticas organizacionais que regulam o processo de trabalho na instituição como um todo;

c - Sobrecarga de Trabalho - percepção dos indivíduos quanto às demandas de produção excessivas definidas para o grupo de empregados;

d - Suporte Material - percepção dos indivíduos sobre a disponibilidade, adequação, suficiência e qualidade dos recursos materiais e financeiros fornecidos pela organização para ajudar a execução eficaz das tarefas;

e - Suporte Social no Trabalho - percepção dos indivíduos acerca da existência e disponibilidade do apoio social e da qualidade do relacionamento interpessoal com a chefia e com os colegas;

f - Ascensão e Salários - percepção dos indivíduos sobre as práticas específicas de retribuição financeira, promoção e ascensão funcionais.

Escala de Coping Ocupacional. Esta escala é a versão brasileira (tradução e adaptação de Pinheiro, Tamayo \& Tróccoli, 2000a; Pinheiro, Tamayo \& Tróccoli, 2000b) da escala proposta por Latack (1986). A escala apresenta os fatores controle $(\alpha=0,79)$; escape $(\alpha=0,77)$ e manejo de sintomas $(\alpha=0,81)$.

O Controle consiste em ações e reavaliações cognitivas proativas; o Escape são ações e reavaliações cognitivas que sugerem fuga ou um modo de evitação e o Manejo de Sintomas são estratégias popularmente aceitas utilizadas pelo indivíduo para administrar eventos relacionados ao estresse, tais como o relaxamento ou a atividade física.

\section{Resultados}

Uma análise preliminar dos dados foi realizada para identificar valores extremos e dados faltosos. Os dados faltosos foram substituídos pela média das variáveis. Foram identificados 27 casos extremos multivariados por meio da distância Mahalanobis $(\alpha=0,001)$. Esses casos não foram considerados nas análises.

A Tabela 1 apresenta a média e o desvio padrão de cada um dos fatores dos três instrumentos utilizados nesta 
Tabela 1. Média, desvio padrão e correlações entre os

fatores das escalas de Percepção de Suporte

Organizacional (PSO) e de Coping no Trabalho e as

dimensões da escala de Exaustão Emocional

\begin{tabular}{|c|c|c|c|c|}
\hline Variáveis & $\mathrm{M}$ & d.p. & $\begin{array}{l}\text { Exaustão } \\
\text { Psicológica }\end{array}$ & $\begin{array}{c}\text { Percepção } \\
\text { de Desgaste }\end{array}$ \\
\hline \multirow{2}{*}{\multicolumn{5}{|c|}{$\begin{array}{l}\text { Escala de ExaustãoEmocional } \\
\text { Exaustão }\end{array}$}} \\
\hline & & & & \\
\hline Psicológica & 2,55 & 0,88 & & \\
\hline Percepção de & & & & \\
\hline Desgaste & 2,07 & 0,76 & $0,64 * *$ & \\
\hline \multicolumn{5}{|l|}{ Escala de PSO } \\
\hline Estilo de Gestã & & & & \\
\hline $\begin{array}{l}\text { Da Chefia } \\
\text { Gestão de }\end{array}$ & 3,30 & 0,80 & $-0,36^{* *}$ & $-0,25^{* *}$ \\
\hline Desempenho & 3,21 & 0,63 & $-0,26^{* *}$ & $-0,10^{*}$ \\
\hline $\begin{array}{l}\text { Sobrecarga } \\
\text { Suporte }\end{array}$ & 2,84 & 0,67 & $0,47 * *$ & $0,51 * *$ \\
\hline $\begin{array}{l}\text { Material } \\
\text { Ascensão e }\end{array}$ & 3,59 & 0,62 & $-0,13 * *$ & $-0,12 *$ \\
\hline Salários & 2,61 & 0,86 & $-0,26^{* *}$ & $-0,14^{* *}$ \\
\hline $\begin{array}{l}\text { Suporte Social } \\
\text { no Trabalho }\end{array}$ & 3,43 & 0,73 & $-0,32 * *$ & $-0,24 * *$ \\
\hline \multicolumn{5}{|c|}{$\begin{array}{l}\text { Escala de Coping_no Trabalho } \\
\text { Maneio de Sintomas }\end{array}$} \\
\hline & 2,26 & 0,70 & 0,03 & 0,06 \\
\hline Controle & 3,78 & 0,50 & $-0,11^{*}$ & $-0,06$ \\
\hline Escape & 2,23 & 0,61 & $0,25 * *$ & $0,18 * *$ \\
\hline
\end{tabular}

pesquisa. Esta tabela também mostra as correlações dos fatores das escalas de PSO e de coping com os fatores da Escala de Exaustão Emocional.

A correlações de Pearson da Tabela 1 mostram que quase todas as dimensões da Escala de Percepção de Suporte Organizacional apresentaram correlações negativas e significativas com as dimensões da Escala de Exaustão Emocional. A dimensão sobrecarga foi a única que apresentou correlações positivas e igualmente significativas com a exaustão psicológica $(0,47)$ e percepção de desgaste $(0,51)$.

Em relação às dimensões da Escala de Coping no Trabalho, pode se observar que o fator escape apresentou uma correlação positiva e significativa com as duas dimensões da Escala de Exaustão Emocional, enquanto que o fator controle só evidenciou uma correlação negativa e, também significativa, com a dimensão exaustão psicológica. O fator manejo de sintomas da Escala de Coping não apresentou nenhuma correlação significativa com as dimensões da Escala de Exaustão Emocional.

Foram realizadas duas análises de regressão múltipla padrão para verificar o poder de predição das dimensões da percepção de suporte organizacional e do coping no trabalho para as dimensões da exaustão emocional

A primeira regressão múltipla, calculada para a dimensão Exaustão Psicológica (Tabela 2), mostra que 31\% da variância dessa dimensão é explicada pelo conjunto de variáveis de suporte organizacional e de coping ocupacional. No entanto, deve-se observar que, considerando o grupo dessas variáveis preditoras, apenas quatro das seis dimensões da Escala do Suporte Organizacional e uma das três dimensões da Escala de Coping contribuíram significativamente para a variância explicada.

$\mathrm{Na}$ segunda regressão múltipla, realizada para a dimensão Percepção de Desgaste (Tabela 3), pode-se observar que $26 \%$ da variância dessa dimensão também é predita pelo conjunto das dimensões de suporte organizacional e de coping no trabalho. Entretanto, apenas as dimensões suporte social no trabalho e sobrecarga da Escala de Suporte Organizacional contribuíram significativamente para explicar essa variância.

Considerando a finalidade e a limitação do presente artigo, não foram realizadas análises por categorias 
Tabela 2. Regressão linear múltipla padrão dos fatores das escalas de Percepção de Suporte Organizacional e de Coping no Trabalho para a dimensão Exaustão Psicológica da escala de Exaustão Emocional

\begin{tabular}{|c|c|c|c|c|}
\hline \multicolumn{2}{|l|}{ Variáveis } & $\begin{array}{c}\text { Exaustão } \\
\text { Psicológica } \\
\text { V.D. }\end{array}$ & B & $\beta$ \\
\hline \multicolumn{5}{|c|}{$\begin{array}{l}\text { Escala de Percepção } \\
\text { de Suporte Organizacional }\end{array}$} \\
\hline \multicolumn{2}{|c|}{ Gestão de Desempenho } & $-0,26$ & $-0,175^{*}$ & $-0,13$ \\
\hline \multicolumn{2}{|c|}{ Estilos de Gestão da Chefia } & $-0,36$ & $-0,016$ & $-0,01$ \\
\hline \multicolumn{2}{|c|}{ Ascensão e Salários } & $-0,26$ & $-0,136^{* *}$ & $-0,13$ \\
\hline \multicolumn{2}{|c|}{ Suporte Social no Trabalho } & $-0,32$ & $-0,190 * *$ & $-0,16$ \\
\hline \multicolumn{2}{|c|}{ Suporte Material } & $-0,13$ & 0,107 & 0,07 \\
\hline \multicolumn{2}{|c|}{ Sobrecarga } & $-0,47$ & $0,467 * *$ & 0,35 \\
\hline \multicolumn{5}{|c|}{ Escala de Coping no Trabalho } \\
\hline \multicolumn{2}{|c|}{ Manejo de Sintomas } & 0,03 & $-0,075$ & $-0,06$ \\
\hline \multicolumn{2}{|c|}{ Controle } & $-0,11$ & $-0,090$ & $-0,05$ \\
\hline \multicolumn{2}{|l|}{ Escape } & 0,25 & $0,255^{* *}$ & 0,18 \\
\hline Média & 2,55 & & & \\
\hline \multirow[t]{2}{*}{ Desvio Padrão } & 0,88 & & & \\
\hline & & & $\begin{array}{l}\mathrm{R}^{2}=0,33 \\
\mathrm{R}^{2} \text { ajustado } \\
\mathrm{R}=0,57^{* *}\end{array}$ & $=0,31$ \\
\hline
\end{tabular}

ocupacionais nem por ramo de empresa, sendo estas objeto de futuras publicações.

\section{Discussão}

As correlações obtidas entre as dimensões da exaustão emocional e os fatores da Escala de Percepção de Suporte Organizacional denotam a importância de variáveis da organização e dos seus agentes na ocorrência de fenômenos ligados à saúde mental no trabalho.

Variáveis de suporte organizacional que envolvem processos de gestão e gerenciamento da chefia podem diminuir a exaustão emocional. Para isso, esses processos devem ser executados de forma participativa e colaboradora, preocupando-se em incentivar e valorizar o trabalho dos subordinados. A exaustão emocional também pode ser reduzida melhorando o suporte social no trabalho por parte de colegas e supervisores e regulando o processo de trabalho da organização através de políticas claras de divulgação de informação, atualização e planejamento.

A implementação de políticas de ascensão, promoção e salários dentro da organização também pode ter efeitos redutores sobre a exaustão emocional.
As altas correlações obtidas entre as dimensões da exaustão emocional e o fator sobrecarga da Escala de Percepção de Suporte Organizacional e, o fato de que esse fator evidenciou-se como um forte preditor para as dimensões da exaustão emocional, corroboraram o vínculo entre esses dois construtos, relatado de forma consistente na literatura sobre burnout (Cordes \& Dougherty, 1993; Gil-Monte \& Peiró, 1997; Maslach \& Jackson, 1986; Schaufeli \& Enzmann, 1998).

No presente estudo, verificou-se uma predominância de variáveis de percepção de suporte organizacional na correlação e na predição da exaustão emocional em comparação com variáveis de coping. Essa constatação reforça o argumento de Aiken e Sloane (1997), acerca de que o burnout está mais relacionado a características do ambiente de trabalho do que a características do trabalhador.

Os resultados referentes às estratégias de coping no contexto ocupacional, especificamente, a evidência de que o uso de estratégias de escape incrementa a exaustão emocional, corroboraram os achados de Leiter (1991). Essa evidência pode ser de utilidade para a prevenção da 
Tabela 3. Regressão linear múltipla padrão dos fatores das escalas de Percepção de Suporte Organizacional e de Coping no Trabalho para a dimensão Percepção de Desgaste da escala de Exaustão Emocional

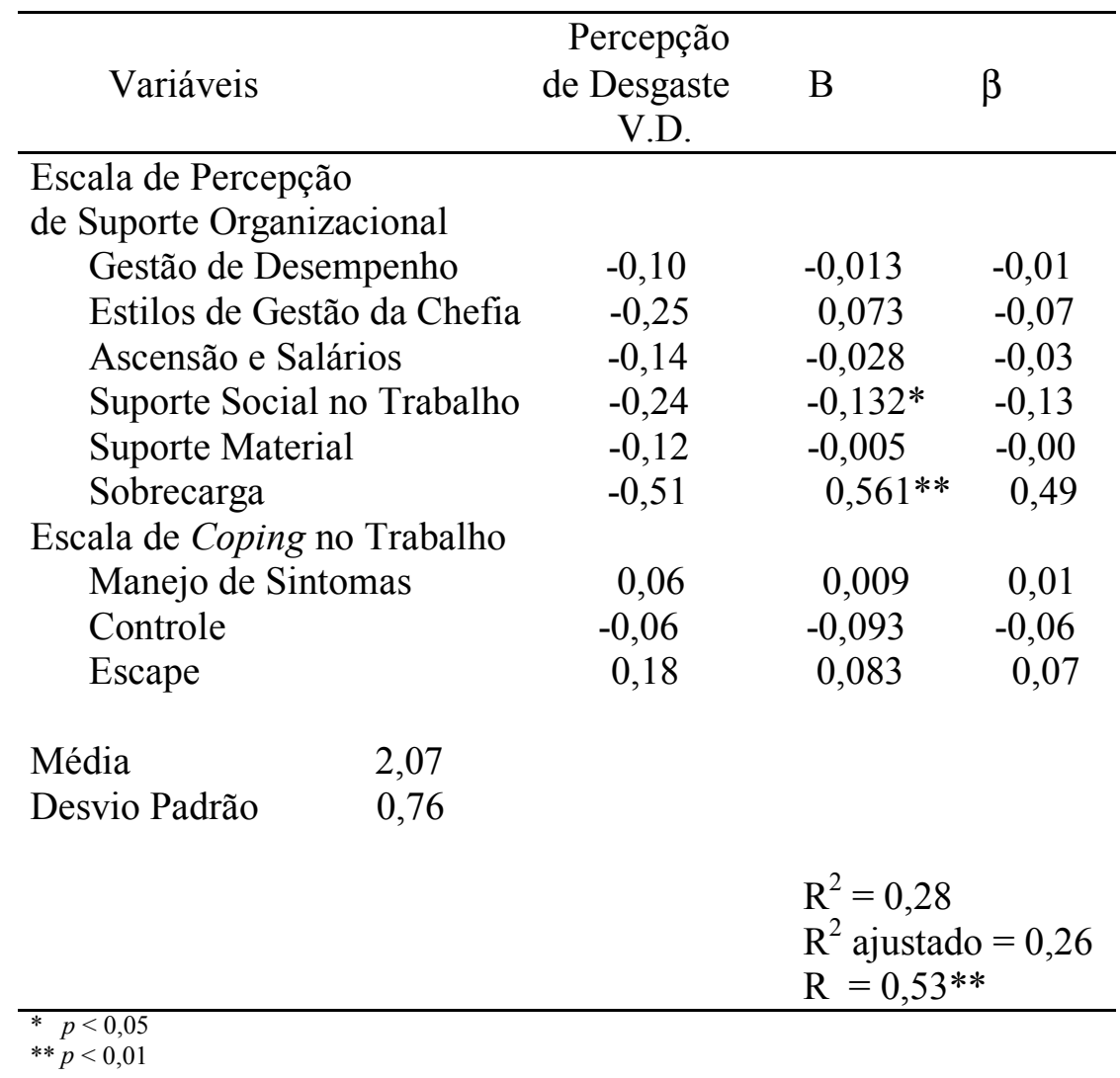

exaustão emocional, através da implementação de programas que levem os sujeitos a recuperar o controle quando lidam com estressores ocupacionais. Neste sentido, o uso de técnicas de reavaliação cognitiva e de relaxamento têm sido implementadas com sucesso para reduzir a exaustão emocional no trabalho (Dierendonck, Schaufeli \& Buunk, 1998).

Intervenções para resolver o burnout e/ou prevenilo recomendam focalizar as soluções tanto no trabalhador quanto no local de trabalho, com a finalidade de desenvolver um processo que permita recuperar o equilíbrio entre as expetativas do indivíduo e as exigências do seu trabalho (Maslach \& Leiter, 1997).

De acordo com esses autores, um programa de prevenção ou de solução do problema do burnout que se concentre exclusivamente no indivíduo, não poderá intervir sobre os aspectos situacionais próprios do contexto social do local de trabalho. Por tal razão, é preciso que os programas levem em conta o vínculo indivíduo-trabalho.
Tanto o estresse ocupacional quanto o burnout e a exaustão emocional são manifestações de tensão no trabalho e são produtos da interação entre fatores ambientais, percepções e comportamentos do indivíduo. Estas manifestações não residem isoladamente dentro do ambiente ou dentro do indivíduo, mas são o resultado de transações dinâmicas que ocorrem entre esses elementos (Cooper, Dewe \& O’Driscoll, 2001).

Nesse sentido, novas pesquisas devem ser desenvolvidas no Brasil para continuar explorando a influência de variáveis que envolvem processos de transação entre o indivíduo e o ambiente, com a finalidade de estabelecer a sua participação no desenvolvimento de fenômenos que atingem a saúde do trabalhador, tais como o estresse ocupacional, o burnout e a exaustão emocional.

\section{Referências}

Aiken. L. H., \& Sloane, D.M. (1997). Effects of organizational innovations in aids care on burnout among urban hospital nurses. Work and Occupations, 24, 453-477. 
Babakus, E., Cravens, D.W., Johnston, M., \& Moncrief, W.C. (1999). The role of emotional exhaustion in sales force attitude and behavior relationship. Academy of Marketing Science Journal, 27, 58-70.

Cooper, C. L., Dewe, P. J., \& O’Driscoll, M. P. (2001). Organizational stress: A review and critique of theory, research, and applications. London: Sage.

Cordes, C. L., \& Dougherty, T. W. (1993). A review and a integration of research on job burnout. Academy of Managemente Review, 18, 621-656.

Dierendonck, D. V., Schaufeli, W. B., \& Buunk, B. P. (1998). The evaluation of an individual burnout intervention program: the role of inequity and social support. Journal of Applied Psychology, 83, 392-407.

Eisenberger, R., Huntington, R., Hutchison, S., \& Sowa, D. (1986). Perceived organizational support. Journal of Applied Psychology, 71, 500-507.

Etzion, D., \& Pines, A. (1986). Sex and culture in burnout and coping among human service professionals: a psychological perspective. Journal of Cross-Cultural Psychology, 17, 191-209.

Folkman, S., Lazarus, R. S., Dunkel-Schetter, C., DeLongis, A., \& Gruen, R. J. (1986). Dynamics of a stressful encounter: cognitive appraisal, coping, and encounter outcomes. Journal of Personality and Social Psychology, 50, 992-1003.

Gaines, J., \& Jermier, J. M. (1983). Emotional exhaustion in a high stress organuizations. Academy of Management Journal, 26, 567-586.

Gil-Monte, P., \& Peiró, J. M. (1997). Desgaste psíquico en el trabajo: el sindrome de quemarse. Madrid: Síntesis.

Jayaratne, S., Himle, D., \& Chess, W. A. (1988). Dealing with work stress and strain: Is the perception of support more important than its use? The Journal of Applied Behavioral Science, 24, 191-202.

Koeske, G. F., Kirk, S. A., \& Koeske, R. D. (1993). Coping with job stress: Which strategies work best? Journal of Occupational and Organizational Psychology, 66, 319-335.

Latack, J. C. (1986). Coping with job stress: measures and future directions for scale development. Journal of Applied Psychology, 71, 377-385.

Latack, J. C., \& Havlovic, A. J. (1992). Coping with job stress: A conceptual evaluation framework for coping measures. Journal of Organizational Behaviuour, 13, 479-508.

Lee, R. T., \& Asforth, B. E. (1996). A meta-analitic examination of the correlates of the three dimensions of job burnout. Journal of Applied Psychology, 81, 123-133.

Leiter, M. (1991). Coping patterns as predictors of burnout: the function of control and escapist coping patterns. Journal of Organizational Behaviour, 12, 123-144.

Levinson, H. (1965). Reciprocation: the relationship between man and organization. Administrative Science Quarterly, 9, 370-390.

Maslach, C. (1993). Burnout: A multidimensional perspective. In W. B. Schaufeli, C. Maslach \& T. Marek (Orgs.), Professional burnout: Recent developments in theory and research (pp.19-32). Washington: Taylor \& Francis.

Maslach. C., \& Jackson, S. (1986). Maslach Burnout Inventory Manual. Palo Alto: Consulting Psychological Press.

Maslach, C., \& Leiter, M. (1997). The truth about burnout: How organizations cause personal stress and what to do about it. San Francisco: Josey-Bass.

Parkes, K. R. (1994). Personality and coping as moderator of work stress process: Models, methods and measures. Work \& Stress, 8, 110-129.
Pinheiro, F., Tamayo, M. R., \& Tróccoli, B. (2000a). Mensuração de coping no ambiente ocupacional: tradução e validação da escala de Latack. [Resumo]. In Sociedade Brasileira para o Progresso da Ciência (Org.), $52^{a}$ Reunião Anual da SBPC, Resumos (CD-ROM). Brasília: SBPC.

Pinheiro, F., Tamayo, M. R., \& Tróccoli, B. (2000b). Mensuração de coping no ambiente ocupacional. Manuscrito submetido para publicação.

Russell, D. W., Altmaier, E., \& Van Velzen, D. (1987). Job-related stress, social support, and burnout among classroom teachers. Journal of Applied Psychology, 72, 269-274.

Schaufeli, W. B., \& Enzmann, D. (1998). The burnout companion to study \& practice: A critical analysis. Philadelphia: Taylor \& Francis.

Schein, E. H. (1982). Psicologia Organizacional. Rio de Janeiro: PrenticeHall do Brasil.

Tamayo, A., Lima, G. L., Siqueira, K. D., Vargas, K. C., Salomão, M. A., \& Rodriguez, W. A. (2000). Exaustão emocional: a profissão e os valores pessoais como preditores. [Resumo]. In Sociedade Brasileira de Psicologia (Org.), XXX Reunião Anual de Psicologia, Resumos (p. 217). Brasília, DF: SBP.

Tamayo, M. R. (1997). Relação entre a sindrome de burnout e os valores organizacionais no pessoal de enfermagem de dois hospitais públicos. Dissertação de Mestrado, Instituto de Psicologia, Universidade de Brasília, Brasilia.

Tamayo, M. R., \& Tróccoli, B. (2000). Construção e validação de uma versão modificada da subescala de exaustão emocional: reinterpretando o constructo de burnout. [Resumo]. In Sociedade Brasileira para o Progresso da Ciência (Org.), $52^{a}$ Reunião Anual da SBPC, Resumos (CD-ROM). Brasília: SBPC.

Tamayo, M. R., Pinheiro, F., Tróccoli, B., \& Paz, M. G. T. (2000). Construção e validação da escala de suporte organizacional percebido (ESOP) [Resumo]. In Sociedade Brasileira para o Progresso da Ciência (Org.), $52^{a}$ Reunião Anual da SBPC, Resumos (CD-ROM). Brasília: SBPC.

Terry, D. (1994). Determinants of coping: the role of stable and situational factors. Journal of Personality and Social Psychology, 66, 895-910.

Thorton, P. I. (1992). The relation of coping, appraisal, and burnout in mental health workers. The Journal of Psychology, 126, 261-271.

VanYperen, N. W. (1998). Informational support, equity and burnout: The moderating effect of self-efficacy. Journal of Occupational and Organizational Psychology, 71, 29-33.

Wright, T. A., \& Bonett, D.G. (1997). The contribution of burnout to work performance. Journal of Organizational Behaviour, 18, 491-499.

Wright, T. A., \& Cropanzano, R. (1998). Emotional exaustion as a predictor of job performance and voluntary turnover. Journal of Applied Psychology, 83, 486-493.

Zautra, A. J., \& Wrabetz, A.B. (1991). Coping success and its relatioship to psychological distress for older adults. Journal of Personality and Social Psychology, 61, 801-810.

Zohar, D. (1997). Predicting burnout with a hassle-based measure of role demands. Journal of Organizational Behaviour, 18, 101-115. 
Mauricio Robayo Tamayo, doutor em Psicologia pela Universidade de Brasília, é atualmente professor do Departamento de Psicologia da Universidade Federal do Rio Grande do Norte, Natal (RN). Bartholomeu Tôrres Tróccoli, doutor em Psicologia Social pela University of Wisconsin (EUA), é professor do Departamento de Psicologia Social e do Trabalho da Universidade de Brasília, Brasília (DF).

Endereço para correspondência: Departamento de Psicologia, UFRN, Caixa Postal 1622, 59078-970, Natal, RN. E-mail: [MRT] maurobayo@hotmail.com; [BTT] troccoli@unb.br. 